\title{
The changes of heart rate variability after unilateral stellate ganglion block
}

\author{
Jang Jae Kim, Rack Kyung Chung, Hee Seung Lee, and Jong In Han \\ Department of Anesthesiology and Pain Medicine, Ewha Womans University School of Medicine, Seoul, Korea
}

Background: The effect of the unilateral stellate ganglion block (SGB) on cardiovascular regulation remains controversial. We wished to evaluate the changes in heart rate variability (HRV) after a unilateral stellate ganglion block in patients with head and neck pain in the present study. Methods: Patients with head and neck pain ( $n=$ 89) were studied. HRV was determined before and after a C6 unilateral stellate ganglion block (right-sided SGB, 40; left-sided SGB, 49) using a paratracheal technique with $1 \%$ mepivacaine $(6 \mathrm{ml})$. Results: There were no significant differences in HRV indices before and after right-sided SGB. The log scale of power in the high frequency range (InHF) was increased and ratio of power in the low frequency range (LF) to power in the high frequency range (HF) ratio was decreased after left-sided SGB. Conclusions: These results demonstrated that left-sided SGB increased parasympathetic activities in patients with head and neck pain. (Korean J Anesthesiol 2010; 58: 56 60)

Key Words: Heart rate, Mepivacaine, Stellate ganglion.

\section{Introduction}

Stellate ganglion blocks (SGB) are widely used for pain relief in outpatient clinics due to its many therapeutic indications and easy maneuvering. It is used locally over stellate ganglion territory disorders in the craniocervical (head and neck) or upper limbs and systemically for angina pectoris, psychosomatic disorders, hormonal disorders, or unbalanced sympathetic nervous system disorders [1]. Local anesthetic SGBs affect patient cardiovascular status through effects on the autonomic nervous system and can induce changes in heart rate. A previous animal experimental study reported that right stellectomy induced reduction of cardioacceleration on motion [2]. Another study demonstrated differences between left and right blocks and their effects on heart rate; the right stellate ganglion block (RSGB) decreased heart rate, while the left stellate ganglion block (LSGB) did not affect the rate [3]. Similarly, long QT syndrome (LQTS) patients who underwent LSGB had shorter QT intervals [4]. Conversely, another study suggested that unilateral SGB did not induce significant changes in the cardiovascular system in terms of rest and motion [5], and the effects of unilateral SGB on cardiovascular

Received: September 1, 2009. Revised: 1st, September 16, 2009; 2nd, October 12, 2009. Accepted: October 14, 2009.

Corresponding author: Rack Kyung Chung, M.D., Department of Anesthesiology and Pain Medicine, Ewha Womans University School of Medicine, Mok-6dong, Yangcheon-gu, Seoul 158-710, Korea. Tel: 82-2-2650-5039, Fax: 82-2-2655-2924, E-mail: rkchung@mm.ewha.ac.kr (c) This is an open-access article distributed under the terms of the Creative Commons Attribution Non-Commercial License (http:// creativecommons.org/licenses/by-nc/3.0/), which permits unrestricted non-commercial use, distribution, and reproduction in any medium, provided the original work is properly cited. 
control are currently unclear. Heart rate variability (HRV) is a measure of variations in heart rate, and HRV analysis is based on non-invasive methods of differentiation between sympathetic and parasympathetic cardiovascular activity degrees. Power spectral analysis may adequately assess the activity of the autonomic nervous system and may reflect baroreceptor-evoked changes in the vagus nerve [6-9]. We evaluated the effects of unilateral SGB on the autonomic nervous system in head and neck pain patients through the monitoring of heart rate and HRV.

\section{Materials and Methods}

Adult patients with head and neck pain (65 years old or less) admitted to our pain relief clinic were used in the present study. All participants were carefully counseled about postSGB sequelae and side effects, and all gave informed consent. A total of 20 subjects (5 men and 15 women) participated in the study, with a mean \pm standard deviation (SD) age of 44 \pm 14.8 years. A total of 89 SGB procedures were performed, including both RSGB $(n=40)$ and LSGB $(n=49)$. Participants with coagulation deficiencies, infections, hypertension, arrhythmias, diabetes, and autonomic nervous system disorders were excluded from further analysis.

Patients were at rest in a supine position for 5 minutes for the stellate ganglion block procedure. A low pillow was inserted underneath the shoulders in order to have the neck extended and the lower jaw relaxed. The puncture region was sanitized, and a $25 \mathrm{G}$ needle was inserted via the anterior paratracheal approach and was advanced directly perpendicular to the anterior surface of the sixth cervical vertebral (C6) transverse process. After a negative aspirate for blood and cerebrospinal fluid, the stellate ganglion was blocked by slowly injecting 1\% mepivacaine solution (6 $\mathrm{ml}$ ). The puncture area was compressed with gauze toward the cauda for 5 minutes after the injection, and the patient procedure table was tilted with an elevated head rest. The efficacy of the stellate ganglion blockage was confirmed by the appearance of Horner's sign.

HRV was recorded for 256 seconds at 15 minutes before and after the SGB. An electrocardiogram (ECG) was continuously recorded using an EKG monitor (LXCJ103, LAXTHA, Korea) and the data was analyzed through a data analyzer (CANS3000, LAXTHA, Korea). Heart rate variables were evaluated before and after the SGB.

Common variables were used to compared time domain measures for HRV analysis, including mean heart rate, the standard deviation of the normal sinus to normal sinus (NN) intervals (SDNN), the square root of the mean sum of the square of differences between adjacent normal-to-normal intervals (RMSSD), the standard deviation of differences between adjacent normal-to-normal intervals (SDSD), and the proportion derived by dividing NN50 (the number of interval differences of successive NN intervals greater than $50 \mathrm{~ms}$ ) by the total number of normal-to-normal intervals (pNN50). SDNN indicates the control capacity of the autonomic nervous system, while RMSSD, SDSD, and pNN50 reflect the activity of the parasympathetic nervous system. The measurements were evaluated by the log scale of total frequency (InTF: 0-0.4 Hz), log scale of very low frequency (InVLF: $\leq 0.04 \mathrm{~Hz}$ ), log scale of low frequency (InLF: 0.04-0.15 $\mathrm{Hz}$ ), and log scale of high frequency (InHF: 0.15-0.4 Hz), LF/HF ratio when times series (on the time domain) were transformed into frequency domain methods. TF represents the total distribution of R-R distance variations (particularly variance). This implies that HRV increases if TF increases, and vice versa. HF is the high-frequency power spectrum from 0.15 to $0.40 \mathrm{~Hz}$, and primarily reflects parasympathetic nervous system activity. LF is low-frequency power spectrum from 0.04 to $0.15 \mathrm{~Hz}$, and reflects both the sympathetic and parasympathetic nervous system activity. However, the sympathetic nervous system has greater influence on LF. The LF/HF ratio (the ratio of the low frequency power spectrum components to the high frequency components) represents the balance between the sympathetic and parasympathetic nervous systems; decreases in the LF/HF ratio suggests that balance is deflected to the parasympathetic nervous system. All variables were represented in mean \pm SD values and were analyzed with the paired t-test by the SAS (v. 8.2) statistical program. Statistical significance was defined at $P$ values $<$ 0.05 .

\section{Results}

There were no significant changes in HRV indices (heart rate, InTF, InVLF, InLF, InHF, the LF/HF ratio, SDNN, RMSSD, pNN50, SDSD) before and after the right stellate ganglion block (Table 1). The InHF value (representing parasympathetic nervous system activity) was significantly decreased after left stellate ganglion block. There were no significant changes in heart rate, InVLF, or InTF (demonstrating control capacity of the autonomic nervous system) or in SDNN and LiLF (suggesting more predominant sympathetic influence over the parasympathetic nervous system). There were also no changes in RMSSD (reflecting parasympathetic nervous system activity), pNN50, and SDSD (Table 1).

\section{Discussion}

Stellate ganglion blocks were operated on patients with head and neck pain in the present study. Right stellate ganglion block did not result in any significant changes in HR, InTF, InVLF, InLF, InHF, LF/HF ratio, SDNN, RMSSD, pNN50, and SDSD. Conversely, there was a significant increase in 
Table 1. Effects of Right and Left Stellate Ganglia Block on the Indices of Heart Rate Variability

\begin{tabular}{|c|c|c|c|c|}
\hline & \multicolumn{2}{|c|}{$\mathrm{RSGB}$} & \multicolumn{2}{|c|}{ LSGB } \\
\hline & Before & After & Before & After \\
\hline HR (beats/min) & $75.1 \pm 10.5$ & $73.9 \pm 9.7$ & $75.4 \pm 13.5$ & $73.5 \pm 11.2$ \\
\hline $\operatorname{lnVLF}\left(\mathrm{ms}^{2}\right)$ & $5.2 \pm 1.1$ & $5.2 \pm 1.0$ & $5.2 \pm 1.0$ & $5.3 \pm 1.1$ \\
\hline $\operatorname{lnLF}\left(\mathrm{ms}^{2}\right)$ & $3.9 \pm 1.1$ & $3.7 \pm 1.4$ & $4.3 \pm 1.3$ & $4.2 \pm 1.3$ \\
\hline $\operatorname{lnHF}\left(\mathrm{ms}^{2}\right)$ & $4.3 \pm 1.3$ & $4.3 \pm 1.3$ & $4.2 \pm 1.4$ & $4.6 \pm 1.1^{*}$ \\
\hline $\operatorname{lnTF}\left(\mathrm{ms}^{2}\right)$ & $5.8 \pm 1.0$ & $5.8 \pm 1.0$ & $5.9 \pm 1.1$ & $6.1 \pm 1.4$ \\
\hline LF/HF ratio & $1.0 \pm 0.4$ & $0.9 \pm 0.2$ & $1.1 \pm 0.4$ & $0.9 \pm 0.2^{*}$ \\
\hline SDNN (ms) & $23.5 \pm 13.0$ & $22.8 \pm 13.2$ & $24.2 \pm 14.0$ & $28.0 \pm 20.2$ \\
\hline RMSSD (ms) & $15.0 \pm 9.7$ & $13.4 \pm 9.0$ & $14.3 \pm 9.7$ & $16.4 \pm 11.3$ \\
\hline SDSD (ms) & $20.5 \pm 13.5$ & $20.2 \pm 14.4$ & $21.1 \pm 15.7$ & $25.0 \pm 24.9$ \\
\hline pNN50 (\%) & $86.5 \pm 15.9$ & $89.4 \pm 15.0$ & $86.6 \pm 14.2$ & $84.8 \pm 15.8$ \\
\hline
\end{tabular}

Results are expressed as mean \pm SD values. RSGB: right stellate ganglion block, LSGB: left stellate ganglion block, HR: heart rate, InVLF: log scale of very low frequency, InLF: log scale of low frequency, InHF: log scale of high frequency, InTF: log scale of total power, SDNN: standard deviation of the normal sinus to normal sinus (NN) interval, RMSSD: square root of the mean sum of the square of differences between adjacent NN intervals, SDSD: standard deviation of differences between adjacent NN intervals, pNN50: the proportion derived by dividing NN50 by the total number of NN intervals. ${ }^{*} \mathrm{P}<0.05$ when compared with before SGB.

InHF, suggesting the presence of increased parasympathetic nervous system activity after left stellate ganglion block. Additionally, there was a significant decrease in the LF/HF ratio, which indicated autonomic nervous system balance.

There are several methods used to assess autonomic nervous system effects on the cardiovascular system. The E:I (expiration: inspiration) ratio of heart rate on deep breathing, heart rate response during the Valsalva maneuver, and changes in heart rate due to changes in stature were used to assess cardiovagal function. Measurement of catecholaminergic solution, blood pressure responses on active standing or passive tilting, blood pressure responses during the Valsalva maneuver, the hand-grip test, the cold-stress test, and the microeneurograph (to assess muscle sympathetic nerve activity, or MSNA) were used to assess sympathetic nervous system function.

Heart rate variability ( $\mathrm{HRV}$ ) is a method used to assess autonomic nervous system function and its effects on the cardiovascular system by analyzing a time series of ECGderived beat-to-beat intervals (the most common clinical procedure to assess this). Heart rate typically represents the number of heartbeats per minute; intervals between heartbeats are not typically uniform and tend to fluctuate. They reflect the influence of the autonomic nervous system and homeostatic mechanisms on the sinoatrial/sinus node. $\mathrm{R}$ - $\mathrm{R}$ intervals should be measured beat-by beat and arithmetic manipulations of R-R intervals should be applied to assess HRV $[10,11]$

Sympathetic nervous system innervation to the heart is not equal to the left and right sides, and therefore the effects of stellate ganglion blocking are different as well. Efferent sympathetic nerves from the left stellate ganglion are distributed in the artrioventricular node and the myocardium (posterior to the left ventricle), while efferent sympathetic nerves from the right stellate ganglion are distributed in the sinus node and the myocardium (anterior to the left ventricle). Therefore, if the left stellate ganglion is blocked, then Therefore, the refractory period of the posterior left ventricle is extended if the left stellate ganglion is blocked [3, 12].

The effects of unilateral stellate ganglion block on cardiovascular control are unclear. A previous study suggested that $1 \%$ lidocaine administration to neuralgia patients resulted in a decreased heart rate with right stellate ganglion block and no significant changes in heart rate with left stellate ganglion block [3]. This is likely because the right stellate ganglion branch nerves are densely distributed on the sinus node. A right stellate ganglion block with 1\% lidocaine also decreased heart rate [13]. Han et al. identified a decrease in the heart rate with left stellate ganglion block using 1\% mepivacaine [14]. In contrast, Gardner et al. reported that unilateral stellate ganglion blocks did not influence the cardiovascular system [5]. Fujiki et al. also demonstrated that stellate ganglion block with mepivacaine on either side did not induce any changes in R-R and corrected QT intervals, and that the changes in R-R intervals demonstrated a positive correlation with the change in and amounts of InHF and InTF [15]. These data accordingly suggested that the vagus nerve was located near the stellate ganglion and both the sympathetic nerve and the parasympathetic vagus nerve was blocked. Goh et al. also reported that stellate ganglion block did not result in any changes in blood pressure and heart rate [16]. A study of patients with SGB adaptation syndrome demonstrated that both right and left stellate ganglion blocks did not induce any change in heart rate but did increase blood pressure [17]. Other studies have demonstrated the 
effects of stellate ganglia on heart rates with various results. In contrast, this study did not demonstrate any significant changes in heart rate by either left or right stellate ganglion block.

Fujiki et al. reported that right stellate ganglion block decreased InLF, InHF, and InTF but that left stellate ganglion block did not result in any significant changes in HRV variables [15]. This finding is compatible with right-sided predominance of the autonomic innervation of the sinus mode. However, there were no significant differences after right stellate ganglion block in the present study. In contrast, left stellate ganglion block resulted in an increased InHF and a decreased LF/HF ratio, suggesting parasympathetic nerve activity.

The effects of stellate ganglion block on the autonomic nervous system are usually clinically safe. However, some side effects are associated with ganglion block. Kimura et al. presented a series of serious blood pressure elevations where systolic arterial pressure exceeded $200 \mathrm{mmHg}$ after stellate ganglion block, likely due to local anesthetic infiltration into adjacent tissues resulting in partial block of the vagus nerve and sympathetic accentuation to trigger an increase in blood pressure [18]. Ashok et al. reported cases of sinus arrest after stellate ganglion block, and suggested that sympathetic nerve block after stellate ganglion block resulted in parasympathetic accentuation to trigger asystole [19].

There are several reasons that stellate ganglion block affects the cardiovascular system. Stellate ganglion block will initially affect both the sympathetic and parasympathetic nervous systems based on the degree of block. The intensity of right stellate ganglion blockage of the sympathetic and parasympathetic nervous system will result in heart rate changes and altered activity of the sympathetic and parasympathetic nervous system. Efferent sympathetic innervation from the right stellate ganglion is primarily distributed over the sinus node of the heart, and the influence of the autonomic nervous system and left stellate ganglion block should be assessed. Further, results will be affected by the health status of participants.

Although efferent sympathetic nerves from the stellate ganglion are primarily distributed over the heart, efferent sympathetic fibers from the 2 nd to 5 th thoracic ganglia affect the heart as well. Accordingly, the autonomic nervous system of the heart is not completely dependent on the stellate ganglia. The influence of the autonomic nervous system cannot be excluded as well. It is worth noting that the mepivacaine in the present study was a lower dosage than those used in other studies. Finally, the autonomic nervous system varies in a unique, autonomous manner, and it is therefore difficult to assess changes in patients in a uniform manner or just through the application of one or two indicators. Future studies should examine diverse methods for the assessment of autonomic nervous system function.

Our study did not measure hemodynamic changes, and it is difficult to argue that heart rate variability alone caused sympathetic nerve activation. Unilateral stellate ganglion blocks were performed on patients with head and neck pain and heart rate and HRV were analyzed. Right stellate block did not result in any changes in sympathetic and parasympathetic nervous activity, whereas left stellate block induced increases in parasympathetic nervous system activity. Therefore, the influence of parasympathetic nervous activation on the cardiovascular system must be considered during a stellate ganglion block.

\section{References}

1. Wakusugi B. New application of stellate ganglion block. Korean J Pain 1991; 4: 1-7.

2. Randall WC, Rohse WG. The augmentor action of the sympathetic cardiac nerves. Circ Res 1956; 4: 470-5.

3. Rogers MC, Battit G, McPeek B, Todd D. Lateralization of sympathetic control of the human sinus node: ECG changes of steeate ganglion block. Anesthesiology 1978; 48: 139-41.

4. Schwarz PJ, Locati EH, Moss AJ, Crampton RS, Trazzi R, Rubreti $U$, et al. Left cardiac sympathetic denervation in the therapy of congenital long QT syndrome: a worldwide report. Circulation 1991; 84: 503-11.

5. Gardner MJ, Kimber S, Johnstone DE, Shukla RC, Horacek BM, Forbes C, et al. The effects of unilateral stellate ganglion blockade on human cardiac function during rest and exercise. J Cardiovasc Electrophysiol 1993; 4: 2-8.

6. Akselrod S, Gordon D, Ubel FA, Shannon DC, Barqer $A C$, Cohen RJ. Power spectrum analysis of heart rate fluctuation: A quantitative probe of beat-to-beat cardiovascular control. Science 1981; 213: 220-2.

7. Pomeranz B, Macaulay RJ, Candil MA, Kutz I, Adam D, Gordon D, et al. Assessment of autonomic function in humans by heart rate spectral analysis. Am J Physiol 1985; 248: H151-3.

8. Pagani M, Lombardi F, Guzzetti S, Rimoldi O, Furlan R, Pizzinelli $P$, et al. Power spectral analysis of heart rate and arterial pressure variabilities as a marker of sympathovagal interaction in man and conscious dog. Circ Res 1986; 59: 178-93.

9. Kuo TBJ, Lai CJ, Huang YT, Yang CC. Regression analysis between heart rate variability and baroreflex-related vagus nerve activity in rats. J Cardiovasc Electrophysiol 2005; 16: 864-9.

10. Hwang GS. Anesthesia and autonomic nervous system: is measurement of heart rate variability, blood pressure variability and baroreflex sensitivity useful in anesthesiology specialty? Korean J Anesthesiol 2008; 55: 
265-76.

11. Task Force of the European Society of Cardiology and the North American Society of Pacing and Electrophysiology. Heart rate variability: standards of measurement, physiological interpretation and clinical use. Circulation 1996; 93: 1043-65.

12. Yanowitz F, Preston JB, Abildskov JA. Functional distribution of right and left stellate innervation to the ventricle. Production of neurogenic electrocardiographic changes by unilateral alteration of sympathetic tone. Circ Res 1966; 18: 416-28.

13. Kashima T, Tanaka H, Minagoe S, Toda H. Electrocardiographic changes induced by the stellate ganglion block in normal subjects. J Electrocardiol 1981; 14: 169-74.

14. Han JU, Chung CK, Kim TD, Lee CS, Cha YD, Oh JD, et al. The effect of a left stellate ganglion block on left ventricular function. Korean J Anesthesiol 2000; 39: 798-
803.

15. Fujiki A, Masuda A, Inoue H. Effects of unilateral stellate ganglion block on the spectral characteristics of heart rate variability. Jpn Circ J 1999; 63: 854-8.

16. Goh JS, Min BW, Kim HD. Blood pressure, pulse rate, and temperature changes of the ipsilateral upper extremity after unilateral stellate ganglion block. Korean J pain 1990; 3: 27-33.

17. Kweon TD, Han CM, Kim SY, Lee YW. The changes of blood pressure, heart rate and heart rate variability after stellate ganglion block. Korean J Pain 2006; 19: 202-6.

18. Kimura T, Nishiwaki K, Yokota S, Komatsu T, Shimada Y. Severe hypertension after stellate ganglion block. Br J Anaesth 2005; 94: 840-2.

19. Saxena AK, Saxena N, Aggarwal B, Sethi AK. Unusual complication of sinus arrest following right-sided stellate ganglion block: a case report. Pain Pract 2004; 4: 245-8. 